\title{
Calibrating a homogenized polycrystal model from large scale FE computations of polycrystalline aggregates
}

\author{
Fabrice BARBE, Rodolphe PARISOT, Samuel FOREST, Georges CAILLETAUD \\ Ecole des Mines de Paris/CNRS \\ Centre des Matériaux/UMR 7633, BP 87, 91003 Evry, France \\ Tel : 0160763051 \\ Fax: 0160763150 \\ samuel.forest@mat.ensmp.fr
}

\begin{abstract}
Homogenization-based estimates of the effective behaviour of elastoviscoplastic polycrystals usually resort to approximations like that of an inclusion embedded in the homogeneous equivalent medium in the case of the self-consistent scheme. We propose here to replace this idealized intermediate problem by the actual computation of a polycrystalline representative volume element. Computations of a cube made of 200 zinc grains are presented and used to calibrate the scale transition rule of a simplified homogenized polycrystal model. A coupled identification procedure is presented to determine both intragranular material parameters and scale transition parameters from experimental tension, creep and relaxation tests.
\end{abstract}

\section{INTRODUCTION TO COMPUTATIONAL HOMOGENIZATION METHODS}

Homogenization methods are now a well-established tool of the mechanics of heterogeneous materials to predict the effective properties from the knowledge of the constituents, at least in the linear regime. In elastoplastic and purely viscoplastic cases, bounds and estimates are available, at least for simple loading paths [1]. Estimates like the well-known self-consistent scheme provide the values of the mean stress and strain in each individual phase. In a metal polycrystal, a phase is an ensemble of grains having the same crystallographic orientation up to a given solid angle. According to the self-consistent scheme, the state of each phase is evaluated using the solution of the problem of an inclusion of this phase embedded in the wanted homogeneous equivalent medium (HEM). This latter problem admits no exact analytical solution in the non-linear case and one usually takes a homogeneous tangent or secant operator for the matrix as an approximation [2]. In [3], the authors resort to finite element (FE) computations to solve the inclusion problem and tune some parameters of the homogenized model. The problem of the inclusion embedded in the unknown HEM can also be seen as an implicit problem for the effective properties and an inverse approach can be used to determine the properties of the HEM. In [4] a simple $J_{2}$-plasicity constitutive framework is adopted for the HEM and the finite element method is used iteratively to calibrate the parameters and fulfill the self-consistency condition. A similar inverse problem is solved in [5] and [6] but a more complex form of the constitutive equations is retained for the HEM including explicit scale transition rules [7]. Again, inclusion problems are solved using the FE method to calibrate the parameters involved in the scale transition rule. A scale transition rule is a relation giving the mean stress (or strain) in a phase as a function of the mean macroscopic stress (or strain) and other internal variables. The use of simplified explicit transition rules is illustrated in section 4.

Currently available computing capabilities make it now possible to abandon the approximation of the grain as an inclusion in an infinite matrix and to compute instead a volume element of polycrystal containing enough grains to regard it as representative of the polycrystal. Such computations of polycrystalline representative volume elements can be performed in the three-dimensional case relying on parallel computing [8,9]. This approach provides the full heterogeneous stress and strain fields inside the grains including the grain boundary effects. The mean stress and strain can be computed over the whole volume or over each phase and can be compared to the prediction of simplified homogenization models. In particular such computations can be regarded as reference calculations to assess the quality of new estimations of bounds from homogenization theory. 
In the present work we propose to calibrate simplified homogenization models directly from the prediction of computations of polycrystalline aggregates. Simplified models are still necessary since the computations of polycrystalline aggregates are lengthy and require parallel computing. They should however include no explicit yield surface at the macroscopic level and instead incorporate simplified scale transition rules in order to be able to be able to account for large distorsion of the effective yield surface. We apply this methodology to the case of an elastoviscoplastic material for which accurate estimates remain seldom.

\section{MECHANICAL PROPERTIES OF BULK ZINC}

The elastoviscoplastic behaviour of a zinc alloy used as a coating material in galvanized steel sheets has been investigated at room temperature [10]. It has been possible to produce bulk zinc ingots that have been rolled and from which plate samples have been machined. A heat treatment has been chosen to produce a grain size of about $40 \mu \mathrm{m}$. The material is anisotropic due to the typical zinc rolling texture and only tests in the rolling direction are considered here. Tensile tests at different strain rates, creep and relaxation tests have been performed; some results are given in Fig. 1 together with simulations that will be explained in section 5. Because of its low melting temperature, zinc displays a strongly viscoplastic behaviour at room temperature. Because of the alloying elements, the material is found to be much harder than pure zinc.

Zinc has a closed-packed hexagonal crystallographic structure. Two main slip systems have been found to be activated during the deformation of bulk zinc, namely basal slip on the basal plane (0001) in direction $\underline{a}<11 \overline{2} 0>\left(3\right.$ slip systems) and pyramidal $\Pi_{2}$ slip on plane $\{11 \overline{22}\}$ in direction $\underline{c}+\underline{a}<\overline{11} 23>$ (6 slip systems). These systems are included in the constitutive modelling. The constitutive equations describing the intragranular behaviour of zinc are the following [8] :

$$
\begin{gathered}
\underset{\sim}{\varepsilon}=\underset{\sim}{\varepsilon^{e}}+{\underset{\sim}{\varepsilon}}^{p}, \quad \underset{\sim}{\sigma}=\underset{\sim}{C}: \underset{\sim}{\varepsilon_{\sim}^{e}}, \quad \underset{\sim}{\dot{\varepsilon}^{p}}=\sum_{s=1}^{N} \dot{\gamma}^{s}\left(\underline{m}^{s} \otimes \underline{n}^{s}+\underline{n}^{s}+\underline{m}^{s}\right) / 2 \\
\dot{\gamma}^{s}=\operatorname{Max}\left(0,\left(\frac{\left|\tau^{s}-x^{s}\right|-\tau_{c}^{s}}{K^{s}}\right)^{n^{s}}\right) \operatorname{sign}\left(\tau^{s}-x^{s}\right), \quad \tau^{s}=\underset{\sim}{\sigma}:\left(\underline{m}^{s} \otimes \underline{n}^{s}\right),
\end{gathered}
$$

The analysis is restricted to small strains. The total strain is split into elastic and plastic parts and the stress is computed from Hooke's law (1). A Schmid law is adopted. The slip rate $\dot{\gamma}^{s}$ is given by a Norton type equation with a threshold $\tau_{c}^{s}$. $\tau^{s}$ denotes the resolved shear stress on slip system $s$. The $N$ slip systems include both basal and pyramidal ones. Two hardening variables are introduced : non linear isotropic hardening $\tau_{c}^{s}$ and kinematic hardening $x^{s}$ according to :

$$
\begin{aligned}
& \tau_{c}^{s}=\tau_{0_{c}}^{s}+\sum_{r=1}^{N} Q^{s} h_{r s}\left(1-\exp ^{-b_{r} \gamma_{c u m}^{r}}\right), \quad \dot{\gamma}_{c u m}^{s}=\left|\dot{\gamma}^{s}\right| \\
& x^{s}=c^{s} \alpha^{s}, \quad \dot{\alpha}^{s}=\dot{\gamma}^{s}-d^{s} \alpha^{s}\left|\dot{\gamma}^{s}\right|-\left(\frac{x^{s}}{M^{s}}\right)^{m^{s}} \operatorname{sign}\left(\alpha^{s}\right)
\end{aligned}
$$

The internal variable $x^{s}$ is necessary to better account for the relaxation tests and its evolution law (4) contains dynamic and static recovery terms. The isotropic hardening evolution law (3) describes the non linear evolution of the critical resolved shear stress as a function of cumulated slip $\gamma_{c u m}$ and involves an interaction matrix :

$$
\begin{aligned}
& \text { basal } \pi_{2}
\end{aligned}
$$

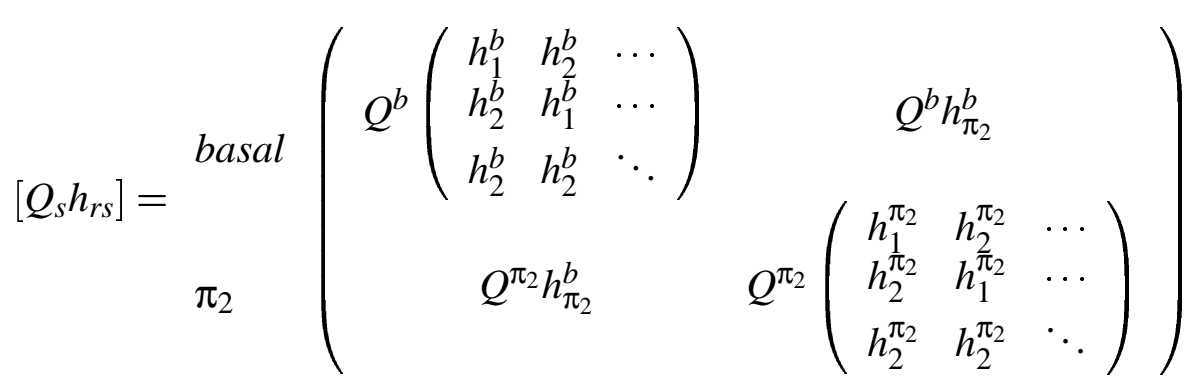


Each slip system familly has its own set of material parameters $K, n, Q, b, c, d, M, m$, but in order to reduce the number of unknowns, a constraint on the ratio of the respective parameters is imposed in the following identification procedure :

$$
Q^{\text {bas }} / Q^{\pi_{2}}=\tau_{0 c}^{\text {bas }} / \tau_{0 c}^{\pi_{2}}=K^{b a s} / K^{\pi_{2}}=\tau_{0 c}^{\text {bas }} / \tau_{0 c}^{\pi_{2}}=c^{b a s} / c^{\pi_{2}}=1 / 5
$$

$n, b, d, M, m$ are the same for all slip systems. Furthermore, we take [10] :

$$
h_{1}^{b}=h_{2}^{b} / 2=h_{1}^{\pi_{2}}=h_{2}^{\pi_{2}} / 2.2=h_{2}^{\pi_{2}} / 2.6
$$

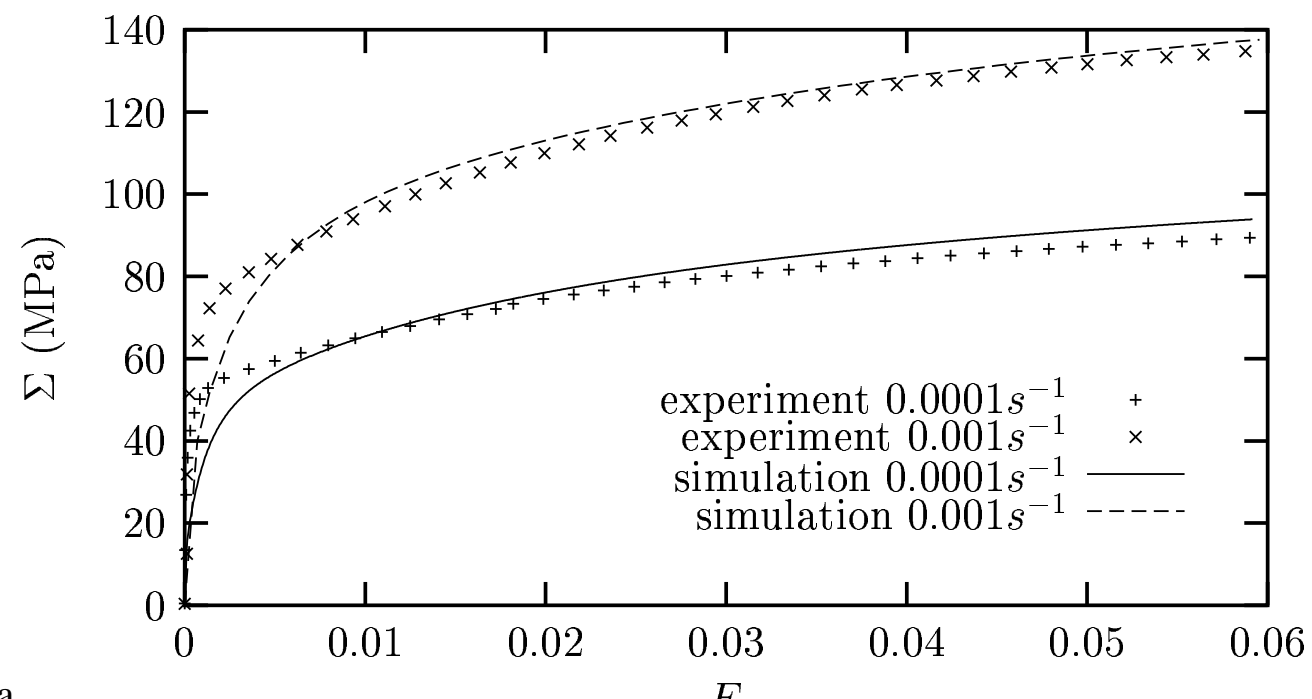

a.
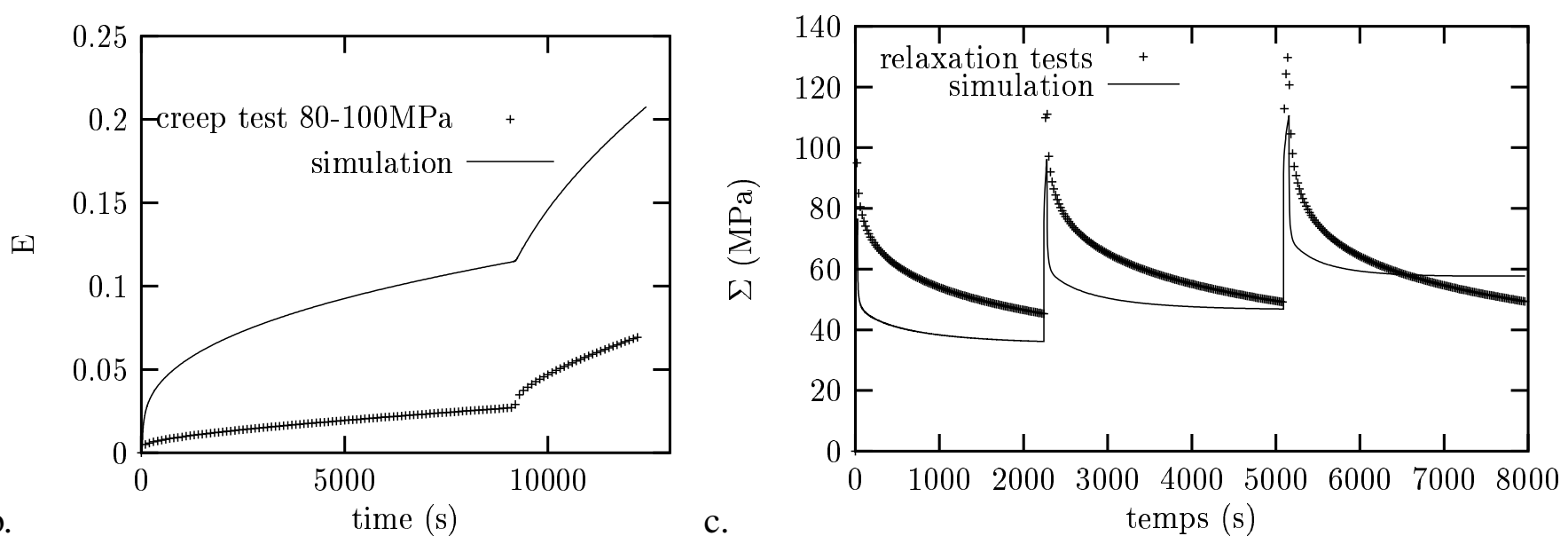

Figure 1. Viscoplastic behaviour of bulk zinc : tensile tests at two strain rates (a); creep test at $80 \mathrm{MPa}$ followed by a jump to $100 \mathrm{MPa}(\mathrm{b})$; relaxation tests at three different strain levels (c).

\section{COMPUTATION OF POLYCRYSTALLINE AGGREGATES}

The three-dimensional distribution of the grains in a sample can be observed experimentally for instance in the case of multicrystalline specimens containing a small number of large grains [11]. In the general case it still remains almost out of experimental reach. This is why the polycrystalline volume element is generated numerically as a distribution of Voronoi polyhedra [9]. A multiphase element technique is used to superpose a finite element mesh on the image of the microstructure : the same crystallographic orientation is attributed to all integration point inside a given grain. In the present work, an isotropic random set of orientations has been randomly distributed among 200 grains. Such a grain distribution and the corresponding mesh are given in Fig. 2a. In the present work, we do not try to represent the anisotropic overall behaviour of the considered material. Furthermore, homogeneous isotropic elasticity is considered, which is of course actually not the case for zinc grains. The observed macroscopic value of Young's modulus in the rolling direction (130000MPa) is retained. The constitutive equations (1) to (5) 
are integrated at each Gauss point. It is essential to have enough elements in each grain in order to have a converged solution in the sense of mesh refinement. This requires at least 1000 integration points per grain. This is why we resort to parallel computing for the computation of polycrystalline aggregates [11]. The RVE computed in this work is a 18x18x18 quadratic brick cube containing 200 grains and divided into 6 subdomains, each subdomain being computed on a single processor.

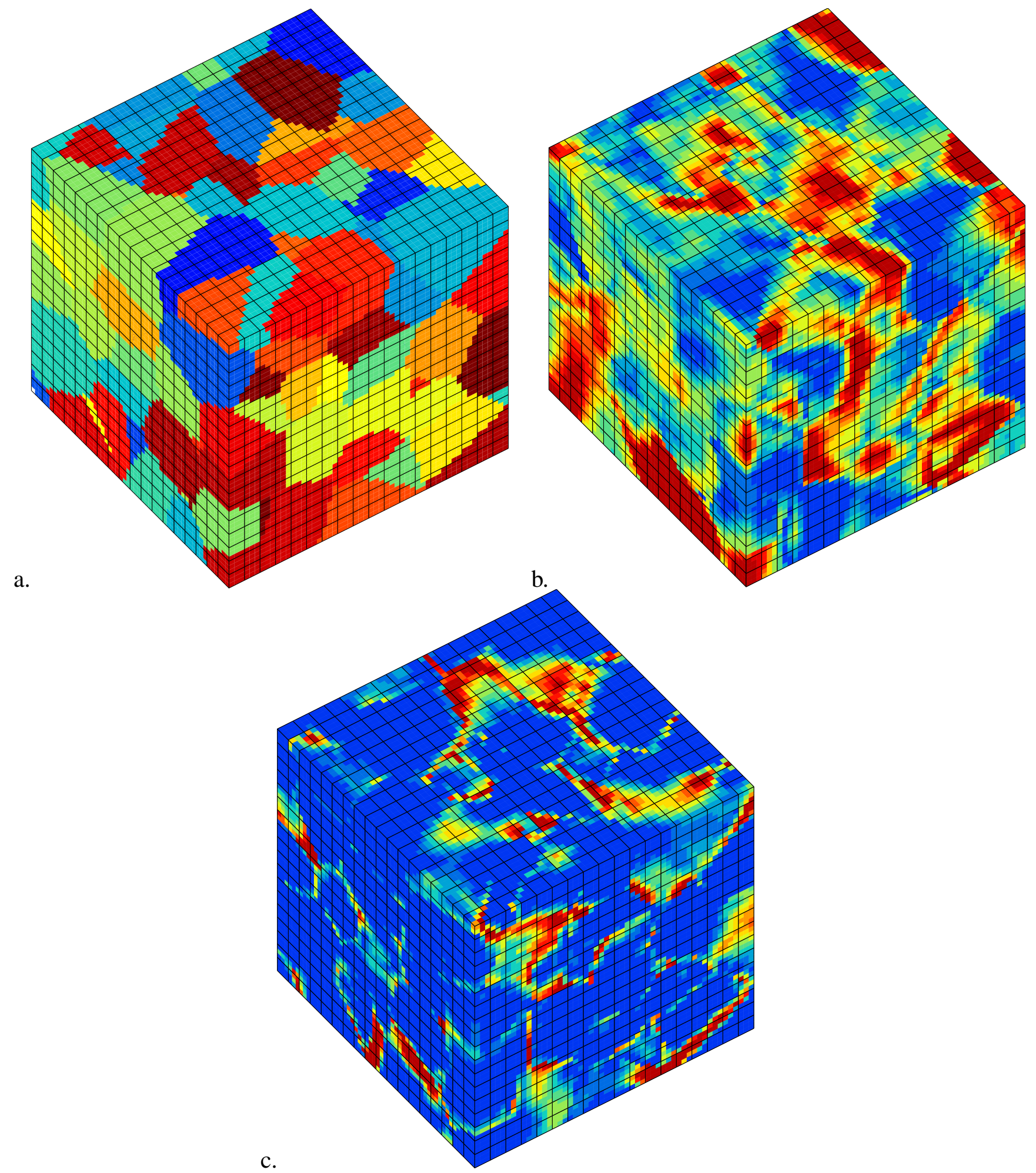

Figure 2. Distribution of 200 grains (a); equivalent basal slip distribution for a mean axial deformation of 0.004 (b, the red color corresponds to an amount of slip greater than 0.01 ); equivalent pyramidal $\Pi_{2}$ slip (c, the red color corresponds to an amount of slip greater than 0.003).

It is clear that the number of grains remains too small for this volume to be really representative. However, in the case of f.c.c. elastoplastic crystals, it has been found sufficient to reach a stabilized response 
in tension when increasing the number of grains [8]. This has not been checked in the case of h.c.p. crystal yet. Moreover some effects due to the application of boundary conditions can be expected. Again in the case of f.c.c. crystals, it has been shown that the boundary layer effect extends over about one grain, provided a fine enough mesh is used, and even less in the case of free surfaces. In the case of 200 grains, boundary layer effects on the overall curves can therefore not be excluded.

Two tensile tests and a shear test have been computed on this aggregate, for a given set of material parameters. For tension, the vertical displacement is prescribed on two opposite faces of the cube, the remaining faces being free of forces. This corresponds to a mean prescribed axial deformation from 0 to $1 \%$. The distribution of basal slip and pyramidal slip activity is given in Fig. $2 \mathrm{~b}$ and $2 \mathrm{c}$ for mean strain $E=0.5 \%$. A very strong heterogeneity is obtained with pyramidal slip occuring mainly at the grain boundaries where plastic incompatibilities develop. This is corroborated by the metallographic observations of Fig. 3 which shows slip lines near some grain boundaries that have been identified as traces of pyramidal slip planes [10]. This is due to the fact that the initial critical resolved shear stress for pyramidal slip is much larger than for basal slip. Note that the observations have been made at a free surface of a specimen and that the view of Fig. 2 also corresponds to free lateral surfaces. The computation gives a similar effect in the core of the aggregate. The mean axial stress $\Sigma$ vs. mean axial strain $E$ curves are given in Fig. 5, for two different tensile strain rates, together with other simulation results to be explained in section 5 .

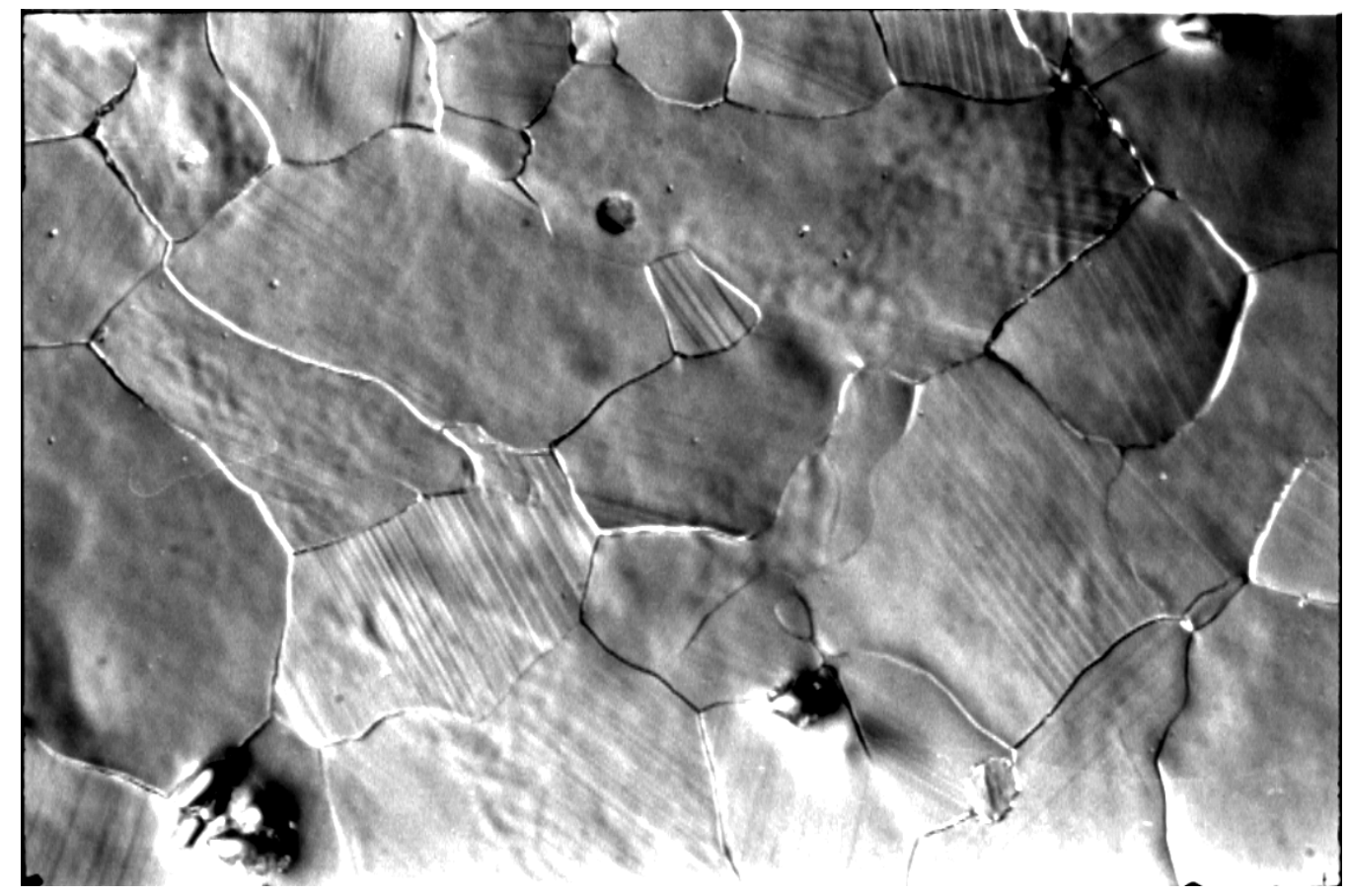

Figure 3. Experimental evidence of basal and pyramidal $\Pi_{2}$ slip in zinc grains (grain size about $40 \mu \mathrm{m}$ ).

\section{EFFECTIVE POLYCRYSTAL MODEL}

Homogenized polycrystal models are available to estimate the mean stress-strain in the phases of the polycrystal in the case of elastoplastic, purely viscoplastic and even elastoviscoplastic grain behaviour, for instance according to the self-consistent scheme [1,2]. In the polycrystal model proposed in [6,7] an explicit scale transition rule is introduced linking the mean local stress ${\underset{\sim}{g}}^{g}$ in grain $g$ to the macroscopic stress $\underset{\sim}{\Sigma}$, the mean viscoplastic deformation $\underset{\sim}{E} p$ and the mean local viscoplastic deformation $\underset{\sim}{p g}$ according to :

$$
\begin{aligned}
& \underset{\sim}{E}=\underset{\sim}{E^{e}}+\underset{\sim}{E}{ }^{p}, \quad \underset{\sim}{\sum}=\underset{\sim}{C}: \underset{\sim}{E^{e}}, \quad \underset{\sim}{\sum_{g}}=\sum_{g=1}^{N_{g}} f_{g}{\underset{\sim}{g}}^{g}, \quad \underset{\sim}{\dot{E}^{p}}=\sum_{g=1}^{N_{g}} f_{g} \dot{\sim}^{p g} \\
& \underset{\sim}{\sigma^{g}}=\sum_{\sim}+C\left(\underset{\sim}{B}-\underset{\sim}{\beta^{g}}\right), \quad \underset{\sim}{B}=\sum_{g=1}^{N_{g}} f_{g}{\underset{\sim}{\beta}}^{g}, \quad \underset{\sim}{\dot{\beta}^{g}}={\underset{\sim}{\dot{\varepsilon}}}^{p g}-D \dot{\varepsilon}_{e q}^{p g}{\underset{\sim}{\beta^{g}}}^{g}
\end{aligned}
$$

where homogeneous elasticity is assumed. The volume fraction of phase $g$ is denoted by $f_{g}, N_{g}$ is the number of discretized orientations (phases). Non linear accommodation variables $\beta^{g}$ are introduced, that 
gradually depart from ${\underset{\sim}{\varepsilon}}^{p g}$ according to the non linear evolution rule in (8). For specific values of parameters $C$ and $D$, classical formulation like Sachs, Lin or Kröner polycrystal models are retrieved. In [5,7], the scale transition parameters $C$ and $D$ have been tuned to mimic the response of the self-consistent scheme. $C$ varies then from 0 to the order of magnitude of the shear modulus. Once the stress $\sigma_{\sim}^{g}$ is computed in each grain, the single crystal constitutive equations (1) to (5) are used to compute the viscoplastic strain rate ${\underset{\sim}{\dot{\varepsilon}}}^{p g}$. In the next section, we will calibrate them to account for the actual response of the aggregate. The question is whether the form of the equations (7) and (8), which is not far from standard homogenization models for elastoplastic polycrystals, will still be suited to describe the elastoviscoplastic behaviour of a zinc aggregate.

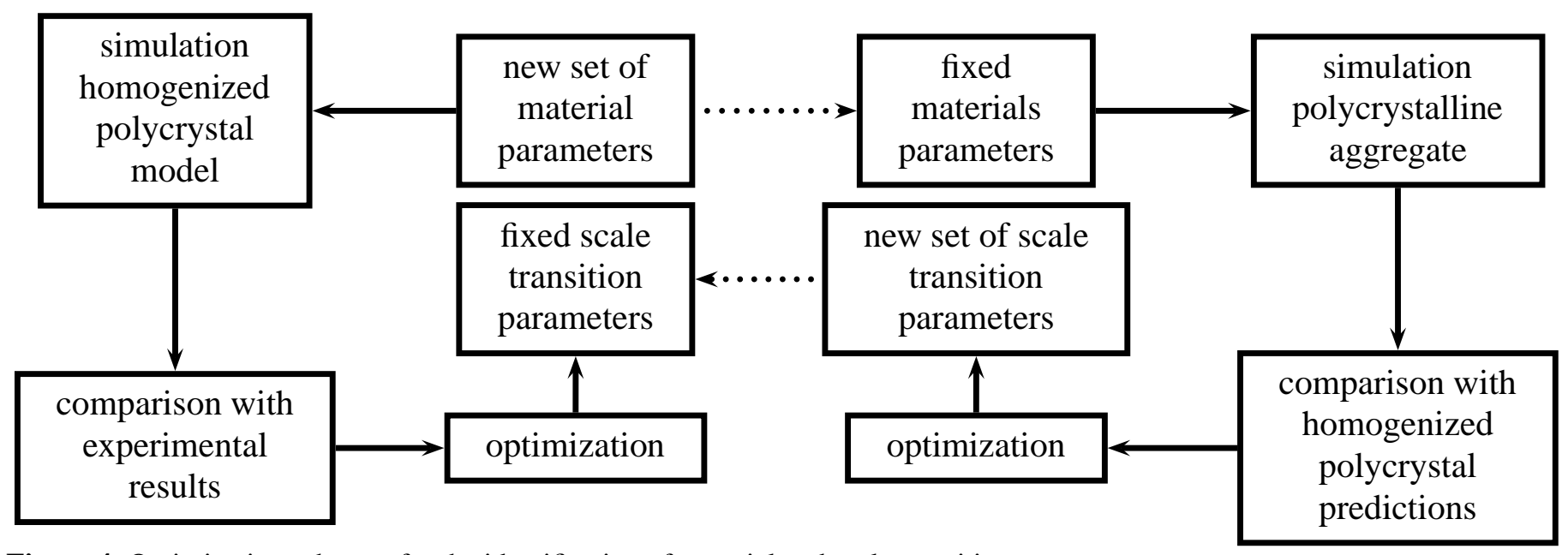

Figure 4. Optimization schemes for the identification of material and scale transition parameters.

\section{OPTIMIZATION PROCEDURE}

Two group of parameters arose in the course of the previous sections : material parameters describing the intragranular behaviour and scale transition parameters appearing in the scale transition rule (8). The second group can be identified using the response of a polycrystalline aggregate with given material parameters. The homogenized model must then be able to reproduce the macroscopic behaviour given in section 2. The identification procedure here requires simulation with both models (aggregate and homogenized polycrystal) and the computation of two cost functions : the first one $\mathcal{C}_{1}$ measures the difference between the mean response of the aggregate and the corresponding simulation with the homogenized model, whereas the second one $C_{2}$ penalizes the difference between this simulation and the experimental curve. An optimization procedure enables one then to find the best set of material and scale transition parameters fulfilling both requirements. In the process of comparing the RVE computation and the homogenized estimate, one should not only check that the macroscopic response is corrected but also that the mean stress/strain in each phase are correctly reproduced by the homogenized model.

A computation with 6 processors of the considered aggregate lasts 5 days whereas a simulation with the homogenized polycrystal model needs a few minutes. This is why a slightly different strategy is adopted represented in Fig. 4. In fact we decouple the two identification procedures. A first trial for $C, D$ is used to optimize the material parameters to minimize $C_{2}$. Once an acceptable fit is obtained, one checks the validity of $C, D$ by computing a polycrystalline aggregate with the new material parameters, which gives $C_{1}$. A new estimation of $C, D$ is then proposed and one goes back to the first iterative procedure. In the present work, we have carried out these three stages only once. A tensile test at $0.001 s^{-1}$ has been used to identify $C, D$, an acceptable fit has been obtained (Fig. 5). As a validation, a tensile test at $0.0001 s^{-1}$ has been simulated with the aggregate and the mean stress-strain curve has been compared to the simulation of the homogenized model. Figure 5 shows that the viscous effect is significantly underestimated by the homogenization model.

The bunch of curves of Fig. $6 a$ and $6 \mathrm{~b}$ gives the mean value of axial stress vs. axial strain for all 200 grains for both models. It appears that stress heterogenity is much more pronounced in the aggregate than 
predicted by the homogenization model. In fact, the number of grains in the aggregate should be large enough to have several representatives of the same given orientations, to be able to compute a mean value over several grains, which is the actual meaning of the phase in the homogenization model. This has been done for f.c.c. crystals and the result is closer to the homogenized solution [9]. In the present case of h.c.p. crystals, four specific grains are compared for both simulations in Fig. 7a. Finally a shear test has been performed on the aggregate using homogeneous deformation conditions at the boundary, at $0.0001 s^{-1}$. The mean shear stress-strain curve is given in Fig. $7 \mathrm{~b}$ and compared to the prediction of the homogenized polycrystal model, which underestimates the attained shear stress levels.

\section{CONCLUSIONS AND PROSPECTS}

At the end of this first attempt of a coupled identification, the description of the macroscopic tests remains rather poor : creep deformation is overestimated and the relaxation is too rapid and too small (Fig. 1). The difficulty of the homogenized model to reproduce the different reponses of the aggregate may have two origins :

- the chosen aggregate is too small and a larger number of grains should be considered;

- the scale transition rule (8) is not sufficient to describe elastoviscoplastic materials : improvements involving 1 to 3 more scale transition parameters are proposed in [7,8] that should be tested.

Finally, a better description of the experimental results could be achieved by relieving the constraint on material parameters (6). Note that the real situation of the zinc alloy requires to take anisotropic local elasticity and the crystallographic texture into account. This will probably require bigger aggregates and a more sophisticated scale transition rule. The methodology will remain the same and the present work is a first attempt in this direction which remains to be significantly improved. Absolute grain size effects are not accounted for in this work, so that the found values of materials and scale transition parameters are bound to one specific grain size. However the method could be extended to size-sensitive crystal plasticity models like [12].

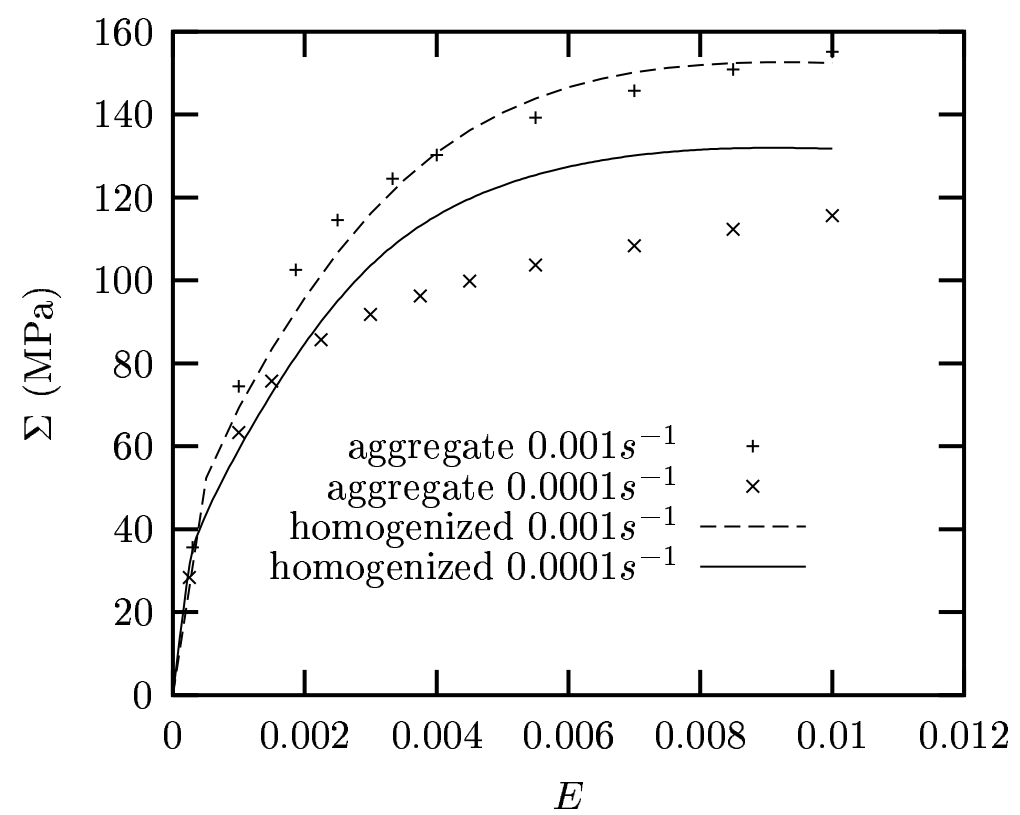

Figure 5. Simulation of tension tests on the polycrystalline aggregate and with the homogenized polycrystalline model. 

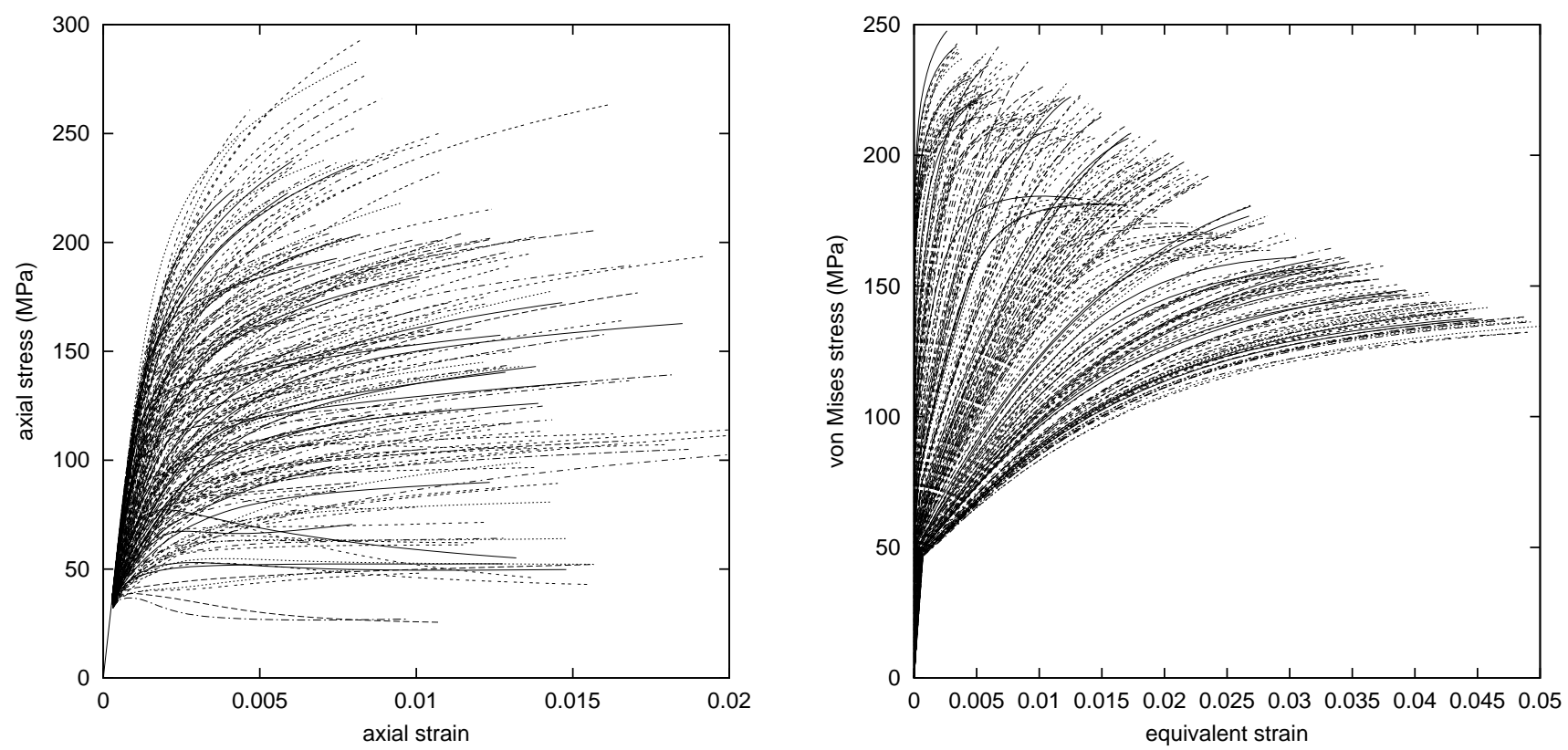

Figure 6. Mean stress-strain states in the 200 grains : aggregate (left) and homogenized model (right).
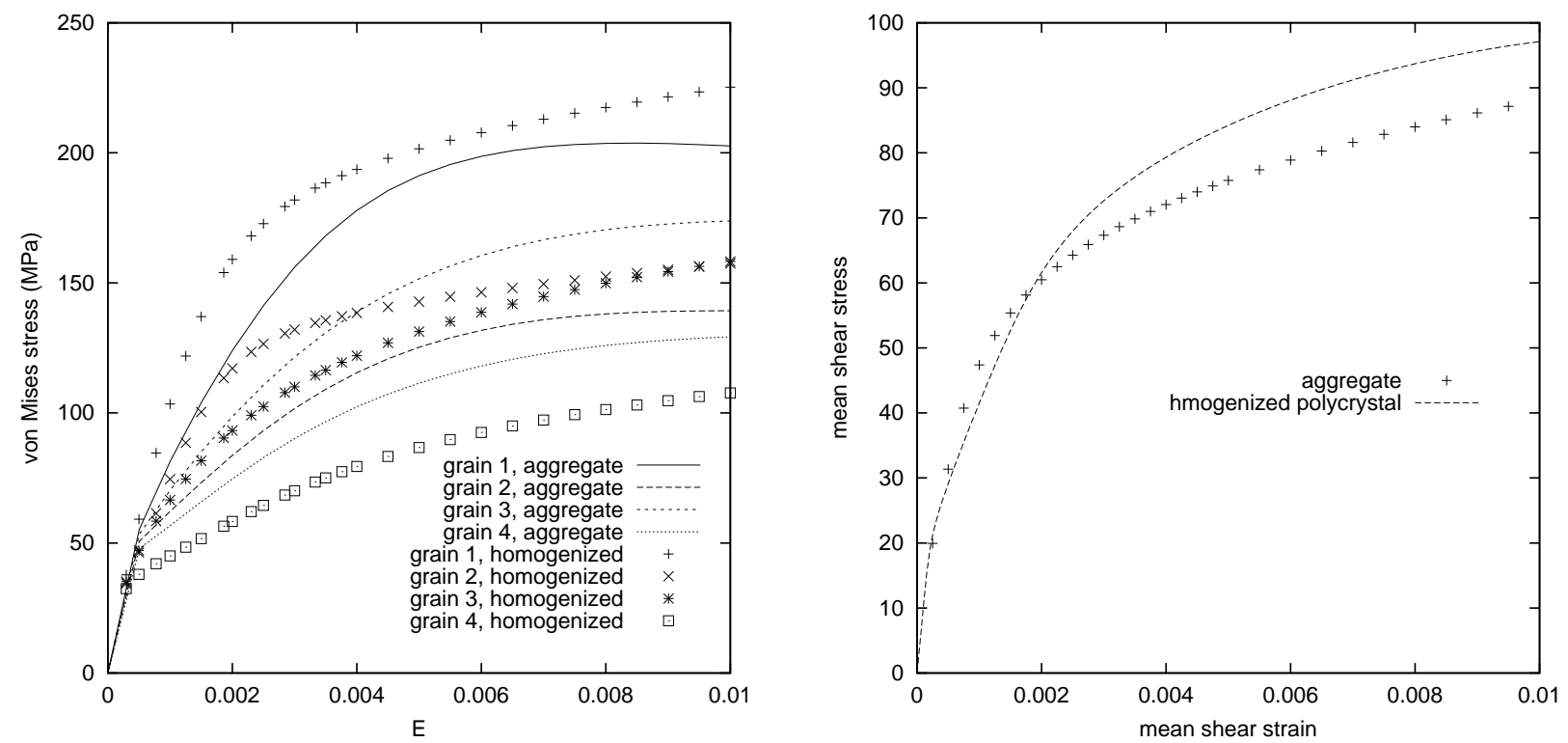

Figure 7. Comparison between the equivalent stress-strain curves obtained by both models for 4 grains (left); overall shear stress-strain curve for the aggregate and prediction of the homogenized model.

\section{References}

[1] Suquet P., Continuum Micromechanics, CISM Courses and lectures No. 397, Springer, 1997.

[2] Molinari A. , Modelling Simulation Mat. Sci. Engng., 7, pp. 683-698, 1999.

[3] Molinari A. and Tóth L., Acta metall. mater, Vol. 42, No. 7, pp. 2453-2458, 1994.

[4] Dong M. and Schmauder S., Acta mater., Vol. 44, No. 6, pp. 2465-2478, 1996.

[5] Forest S. and P. Pilvin, IUTAM Symposium on micromechanics of plasticity and damage of multiphase materials, ed. by A. Pineau and A. Zaoui, Kluwer, pp. 51-58, 1996.

[6] Pilvin P., Multiaxial Fatigue and Design, ESIS 21, ed. by A. Pineau, G. Cailletaud and T. Lindley, Mechanical Engineering Publications, London, pp. 3-19, 1996.

[7] Pilvin P., 3ème Colloque National en Calcul des Structures, 20-23 Mai, Giens, Presses Académiques de l'Ouest, pp. 207-212, 1997.

[8] Quilici S. and Cailletaud G., Computational Materials Science, 16, pp. 383-390, 1999.

[9] Barbe F., Forest S., Cailletaud G. and D. Jeulin, Int. J. Plasticity, in press, 2001.

[10] Parisot R., Forest S., Gourgues A.F., Pineau A. and Mareuse D., Computational Materials Science, 19, pp. 189-204, 2001.

[11] Eberl F., Feyel F., Quilici S. and Cailletaud G., J. Physique IV France, Vol. 8, pp. Pr4-15-25, 1998.

[12] Forest S., Barbe F. and Cailletaud G., Int. J. Solids Structures, Vol. 37, pp. 7105-7126, 2000. 